\title{
Multiple Factorizations of Bivariate Linear Partial Differential Operators
}

\author{
Ekaterina Shemyakova \\ Research Institute for Symbolic Computation (RISC), \\ J.Kepler University, \\ Altenbergerstr. 69, A-4040 Linz, Austria \\ kath@risc.uni-linz.ac.at, \\ WWW home page: http://www.risc.uni-linz.ac.at
}

\begin{abstract}
We study the case when a bivariate Linear Partial Differential Operator (LPDO) of orders three or four has several different factorizations.

We prove that a third-order bivariate LPDO has a first-order left and right factors such that their symbols are co-prime if and only if the operator has a factorization into three factors, the left one of which is exactly the initial left factor and the right one is exactly the initial right factor. We show that the condition that the symbols of the initial left and right factors are co-prime is essential, and that the analogous statement "as it is" is not true for LPDOs of order four.

Then we consider completely reducible LPDOs, which are defined as an intersection of principal ideals. Such operators may also be required to have several different factorizations. Considering all possible cases, we ruled out some of them from the consideration due to the first result of the paper. The explicit formulae for the sufficient conditions for the complete reducibility of an LPDO were found also.
\end{abstract}

\section{Introduction}

The factorization of Linear Partial Differential Operators (LPDOs) is an essential part of recent algorithms for the exact solution for Linear Partial Differential Equations (LPDEs). Examples of such algorithms include numerous generalizations and modifications of the 18th-century Laplace Transformations Method [123/4/5/6 7/8, the Loewy decomposition method 910/11, and others.

The problem of constructing a general factorization algorithm for an LPDO is still an open problem, though several important contributions have been made over the last decades (see for example [9/1213 14215]). The main difficulty in the case of LPDOs is non-uniqueness of factorization: (irreducible) factors and the number of factors are not necessarily the same for two different factorizations of the same operator. For example, for the famous Landau operator [16] $L$ we have $L=\left(D_{x}+1+\frac{1}{x+c(y)}\right) \circ\left(D_{x}+1-\frac{1}{x+c(y)}\right) \circ\left(D_{x}+x D_{y}\right)=\left(D_{x x}+x D_{x y}+\right.$ $\left.D_{x}+(2+x) D_{y}\right) \circ\left(D_{x}+1\right)$. Note that the second order factor in the second factorization is hyperbolic and is irreducible. 
However, for some classes of LPDOs factorization is unique. For example, there is $[9$ no more than one factorization that extends a factorization of the principal symbol of the operator into co-prime factors (see Theorem 1).

Some important methods of exact integration, for example, mentioned above Loewy decomposition methods require LPDOs to have a number of different factorizations of certain types. Also completely reducible LPDOs introduced in [9], which becomes significant as the solution space of a completely reducible LPDO coincides with the sum of those of its irreducible right factors may require a number of right factors. Thus, in Sec. 4 we study the case when a bivariate (not necessarily hyperbolic) LPDO has two different factorizations. For operators of order three we have a really interesting result (Theorems 4 and 5). We showed that analogous statement for operators of order four is not true.

For the proof of the theorems we use invariants' methods. Invariants of LPDOs under the gauge transformations (see Sec. 2) are widely used for factorization problems since Laplace' times as many properties appearing in connection with the factorization of an LPDO are invariant under the gauge transformations, and, therefore, can be expressed in terms of generating invariants, which were found in 17. Factorization itself is invariant under the gauge transformations: if for some LPDO $L, L=L_{1} \circ L_{2}$, then $L^{g}=L_{1}^{g} \circ L_{2}^{g}$. Expressions for necessary and sufficient conditions for the existence of a factorization of a given LPDO of a given factorization type were found in [18 and [19. We use these expressions in the proofs of the theorems of Sec. 5 .

Theorems 4 and 5 of Section 4 allow us to reduce consideration of cases in Sec. 5. where we show how the problem of the complete reducibility of a hyperbolic bivariate LPDO can be expressed in terms of invariants also.

\section{Definitions and Notations}

Consider a field $K$ of characteristic zero with commuting derivations $\partial_{x}, \partial_{y}$, and the ring of linear differential operators $K[D]=K\left[D_{x}, D_{y}\right]$, where $D_{x}, D_{y}$ correspond to the derivations $\partial_{x}, \partial_{y}$, respectively. In $K[D]$ the variables $D_{x}, D_{y}$ commute with each other, but not with elements of $K$. For $a \in K$ we have $D_{i} a=a D_{i}+\partial_{i}(a)$. Any operator $L \in K[D]$ has the form $L=\sum_{i+j=0}^{d} a_{i j} D_{x}^{i} D_{y}^{j}$, where $a_{i j} \in K$. The polynomial $\operatorname{Sym}(L)=\sum_{i+j=d} a_{i j} X^{i} Y^{j}$ in formal variables $X, Y$ is called the (principal) symbol of $L$.

Below we assume that the field $K$ is differentially closed unless stated otherwise, that is it contains solutions of (non-linear in the generic case) differential equations with coefficients from $K$.

Let $K^{*}$ denote the set of invertible elements in $K$. For $L \in K[D]$ and every $g \in K^{*}$ consider the gauge transformation $L \rightarrow L^{g}=g^{-1} \circ L \circ g$. Then an algebraic differential expression $I$ in the coefficients of $L$ is invariant under the gauge transformations (we consider only these in the present paper) if it is unaltered by these transformations. Trivial examples of invariants are the coefficients of the symbol of an operator. A generating set of invariants is a set using which all possible differential invariants can be expressed. 
Given a third-order bivariate LPDO $L$ and a factorization of its symbol $\operatorname{Sym}(L)$ into first-order factors. In some system of coordinates the operator has one of the following normalized forms:

$$
\begin{aligned}
L & =\left(p(x, y) D_{x}+q(x, y) D_{y}\right) D_{x} D_{y}+\sum_{i+j=0}^{2} a_{i j}(x, y) D_{x}^{i} D_{y}^{j} \\
L & =D_{x}^{2} D_{y}+\sum_{i+j=0}^{2} a_{i j}(x, y) D_{x}^{i} D_{y}^{j} \\
L & =D_{x}^{3}+\sum_{i+j=0}^{2} a_{i j}(x, y) D_{x}^{i} D_{y}^{j}
\end{aligned}
$$

where $p=p(x, y) \neq 0, q=q(x, y) \neq 0, a_{i j}=a_{i j}(x, y)$. The normalized form (1) has symbol $S=p X+q Y$. Without loss of generality one can assume $p=1$. Each of the class of operators admits gauge transformations $L \rightarrow g^{-1} \circ L \circ g$ for $g=g(x, y) \neq 0$.

Remark 1. Recall that since for two LPDOs $L_{1}, L_{2} \in K[D]$ we have $\operatorname{Sym}\left(L_{1}\right.$ 。 $\left.L_{2}\right)=\operatorname{Sym}\left(L_{1}\right) \cdot \operatorname{Sym}\left(L_{2}\right)$, any factorization of an LPDO extends some factorization of its symbol. In general, if $L \in K[D]$ and $\operatorname{Sym}(L)=S_{1} \cdot \ldots \cdot S_{k}$, then we say that the factorization

$$
L=F_{1} \circ \ldots \circ F_{k}, \quad \operatorname{Sym}\left(F_{i} 0=S_{i}, \forall i \in\{1, \ldots, k\},\right.
$$

is of the factorization type $\left(S_{1}\right) \ldots\left(S_{k}\right)$.

We reformulate the famous result of $[9]$ in the new notation:

Theorem 1. [9] Let LPDO L of arbitrary order and in arbitrary number of independent variables have symbol $\operatorname{Sym}(L)=S_{1} \ldots S_{k}$, where $S_{i}$-s are pairwise coprime. Then there exists at most one factorization of $L$ of the type $\left(S_{1}\right) \ldots\left(S_{k}\right)$.

\section{Factorization via Invariants for Hyperbolic Bivariate Operators of Order Three}

Information from this section will be used in the proofs below in the case, where $L$ is hyperbolic operator.

Theorem 2. 20] The following 7 invariants form a generating set of invariants for operators of the form (1): $q, I_{1}=2 q^{2} a_{20}-q a_{11}+2 a_{02}, I_{2}=-q a_{02 y}+a_{02} q_{y}+$ $q^{2} a_{20 x}, I_{3}=a_{10}+2 q_{y} a_{20}+a_{20}^{2} q-a_{11 y}+q a_{20 y}-a_{11} a_{20}, I_{4}=a_{01} q^{2}-3 q_{x} a_{02}+$ $a_{02}^{2}-a_{11 x} q^{2}+a_{11} q q_{x}+q a_{02 x}-a_{02} a_{11} q, I_{5}=a_{00} q+2 a_{02} a_{20 x}-a_{02} a_{10}-a_{01} a_{20} q-$ $\frac{1}{2} a_{11 x y} q+q q_{x} a_{20 y}-a_{11} q a_{20 x}+q q_{y} a_{20 x}+2 q^{2} a_{20} a_{20 x}+q q_{x y} a_{20}+a_{20} a_{11} a_{02}$.

The set of values of these seven invariants uniquely defines an equivalent class of operators of the form (11). Also invariant properties of such operators can be described in terms of the seven invariants. 
Lemma 1. The property of having a factorization (or a factorization extending a certain factorization of the symbol) is invariant.

Proof. Let $L=F_{1} \circ F_{2} \circ \ldots \circ F_{k}$, for some operators $F_{i} \in K[D]$. For every $g \in K^{*}$ we have $g^{-1} \circ L \circ g=\left(g^{-1} \circ F_{1} \circ g\right) \circ\left(g^{-1} \circ F_{2} \circ g\right) \circ \ldots \circ\left(g^{-1} \circ F_{k} \circ g\right)$.

Looking through the formulaes of the next theorem, notice that some conditions are the same for different types of factorizations. In particular, one can pay attention to conditions $\left(A_{1}\right)-\left(D_{1}\right)$. Such correlations will be used in the next section (Sec. 4).

Theorem 3. [18] Given the values of the invariants $q, I_{1}, I_{2}, I_{3}, I_{4}, I_{5}$ (from Theorem (2) for an equivalence class of operators of the form (1). The LPDOs of the class have a factorization of factorization type

$(S)(X Y)$ if and only if

$$
\begin{aligned}
& I_{3} q^{3}-I_{1 y} q^{2}+q_{y} I_{1} q-I_{4}+q I_{1 x}-2 q_{x} I_{1}-3 q I 2=0 \\
& -q^{2} I_{4 y}+1 / 2 q^{3} I_{1 x y}-q I_{4 x}-3 / 2 q^{2} q_{x} I_{1 y}+q^{3} I_{5}+q^{2} I_{1 x x} \\
& -3 / 2 I_{1} q^{2} q_{x y}-2 I_{1} q q_{x x}+5 I_{1} q q_{x} q_{y}+6 I_{1} q_{x}^{2}+3 I_{4} q_{x} \\
& +3 I_{4} q q_{y}-q I_{1} I_{1 x}+I_{1} I_{4}+2 q_{x} I_{1}^{2}-4 I_{1 x} q q_{x}-3 / 2 I_{1 x} q^{2} q_{y} \\
& -2 q^{2} I_{2 x}-q^{3} I_{2 y}+I_{2} q I_{1}+4 I_{2} q q_{x}+2 I_{2} q^{2} q_{y}=0
\end{aligned}
$$

$(S)(X)(Y)$ if and only if (4) \& $-I_{4}+q I_{1 x}-2 q_{x} I_{1}-q I_{2}=0$;

$(S)(Y)(X)$ if and only if (7) \& $\left(C_{1}\right)$;

$(X)(S Y)$ if and only if

$$
\left.\begin{array}{l}
\left(D_{1}\right): \quad q q_{x x}-I_{4}-2 q_{x}=0 \\
-3 / 2 q_{x} q I_{1 y}-q^{3} I_{3 x}+I_{5} q^{2}+1 / 2 q^{2} I_{1 x y}-1 / 2 q q_{y} I_{1 x}+ \\
q_{x} q^{2} I_{3}+2 I_{1} q_{x} q_{y}-1 / 2 I_{1} q_{x y} q-4 q_{x} I_{2}+q I_{2 x}=0 .
\end{array}\right\}
$$

$(X)(S)(Y)$ if and only if (5) \& $\left(B_{1}\right)$;

$(X)(Y)(S)$ if and only if (5) \& $\left(D_{1}\right)$;

$(X Y)(S)$ if and only if

$$
\left.\begin{array}{l}
-q I_{2}+q q_{x} q_{y}+q_{y y} q^{3}-q^{2} q_{x y}+q q_{x x}+I_{3} q^{3}-I_{4}-2 q_{x}^{2}=0 \\
q^{3} I_{5}+q I_{4 x}+1 / 2 q^{3} I_{1 x y}-3 / 2 q^{2} q_{x} I_{1 y}+I_{1} I_{4}+q^{2} I_{2 x}+2 I_{1} q q_{x} q_{y} \\
+2 I_{1} q_{x}^{2}-5 I_{4} q_{x}-1 / 2 I_{1} q^{2} q_{x y}-I_{1} q q_{x x}+I_{4} q q_{y}-1 / 2 I_{1 x} q^{2} q_{y}-4 I_{2} q q_{x} \\
-10 q_{x}^{3}-q^{2} q_{x x x}-q^{4} I_{3 x}+I_{3} q^{3} q_{x}+2 q q_{x}^{2} q_{y}-q^{2} q_{y} q_{x x}+8 q q_{x} q_{x x}=0 .
\end{array}\right\}
$$

$(Y S)(X)$ if and only if

$$
\left.\begin{array}{l}
\left(C_{1}\right): \quad-2 q_{x} I_{1}+q I_{1 x}-I_{4}-2 q I_{2}=0, \\
-q I_{4 y}+1 / 2 q^{2} I_{1 x y}+I_{5} q^{2}-I_{2} I_{1}-q^{2} I_{2 y}+2 q_{y} I_{4}+3 I_{1} q_{x} q_{y}- \\
3 / 2 I_{1} q_{x y} q-1 / 2 q q_{y} I_{1 x}-3 / 2 q_{x} q I_{1 y}=0 .
\end{array}\right\}
$$

$(X S)(Y)$ if and only if

$$
\left.\begin{array}{l}
\left(B_{1}\right): \quad I_{3} q^{2}-q I_{1 y}+q_{y} I_{1}-2 I_{2}=0 \\
-1 / 2 q q_{y} I_{1 x}-3 / 2 q_{x} q I_{1 y}+I_{5} q^{2}-q^{3} I_{3 x}+1 / 2 q^{2} I_{1 x y}+q_{x} q^{2} I_{3} \\
-2 q_{x} I_{2}+q I_{2 x}+3 I_{1} q_{x} q_{y}-3 / 2 I_{1} q_{x y} q-I_{2} I_{1}+2 q_{y}^{2} q_{x} q-2 q_{y} q I_{2} \\
-2 q_{x y} q^{2} q_{y}+2 q_{x y} q q_{x}-2 q_{y} q_{x}^{2} .
\end{array}\right\}
$$


$(Y)(S X)$ if and only if

$$
\left.\begin{array}{l}
\left(A_{1}\right): \quad I_{3}+q_{y y}=0 \\
\quad-q I_{4 y}-3 / 2 q_{x} q I_{1 y}+I_{5} q^{2}+1 / 2 q^{2} I_{1 x y}+2 q_{y} I_{4}-1 / 2 q q_{y} I_{1 x}+ \\
3 I_{1} q_{x} q_{y}-3 / 2 I_{1} q_{x y} q-q^{2} I_{2 y}=0 ;
\end{array}\right\}
$$

$(Y)(X)(S)$ if and only if (6) \& $-q q_{x x}+I_{4}+2 q_{x}^{2}+q I_{2}-q q_{x} q_{y}+q^{2} q_{x y}=0$; $(Y)(S)(X)$ if and only if (7) \& $\left(A_{1}\right)$;

For LPDOs of the forms (2) and (3) generating sets of invariants and the corresponding conditions of the existence of factorizations of different types are know also [17, [19].

\section{Several Factorizations of One Operator}

Theorem 4. Let $\operatorname{gcd}\left(S_{1}, S_{2}\right)=1$. A third-order bivariate operator $L$ has a firstorder left factor of the symbol $S_{1}$ and a first-order right factor of the symbol $S_{2}$ if and only if it has a complete factorization of the type $\left(S_{1}\right)(T)\left(S_{2}\right)$, where $T=\operatorname{Sym}(L) /\left(S_{1} S_{2}\right)$.

The following diagram is an informal illustration of the statement of the theorem:

$$
\left(\left(S_{1}\right)(\ldots) \wedge(\ldots)\left(S_{2}\right)\right) \Longleftrightarrow\left(S_{1}\right)(\ldots)\left(S_{2}\right)
$$

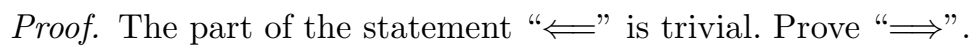

The symbol of the operator has two different factors, therefore, the normalized form of the operator $L$ is either (1) or (2). Without loss of generality we can consider $L$ in its normalized form.

Consider the first (hyperbolic) case. For this class of operators we have the generating system of invariants $q, I_{1}, I_{2}, I_{3}, I_{4}, I_{5}$ from Theorem 2 The symbol is the same for all the operators in the class and is $X \cdot Y \cdot S$, where $S=p X+q Y$. The following six cases are the only possibilities for the $S_{1}$ and $S_{2}, \operatorname{gcd}\left(S_{1}, S_{2}\right)=1$.

Case $S_{1}=S, S_{2}=Y$. By the Theorem 3 operator $L$ has a left factor of the symbol $S_{1}=S$ and a right factor of the symbol $S_{2}=Y$ if and only if conditions (41) and (8) are satisfied. From the second equality in (8) derive an expression for $I_{3}$ and substitute it for $I_{3}$ into the first equality in (4). The resulting equality implies that the third condition (in Theorem 3) for the existence of factorization of the type $(S)(X)(Y)$ is satisfied. The remaining two first conditions are exactly the same as two conditions (44), and therefore the theorem is proved for this case.

Case $S_{1}=Y, S_{2}=S$. Operator $L$ has a left factor of the symbol $S_{1}=Y$ and a right factor of the symbol $S_{2}=S$ if and only if conditions (9) and (6) are satisfied. From the first equality in (9) derive an expression for $I_{3}\left(I_{3}=-q_{y y}\right)$ and substitute it for $I_{3}$ into the first equality in (6). The resulting equality implies that the third condition (in Theorem [3) for the existence of factorization of the type $(Y)(X)(S)$ is satisfied. Since the first two conditions are satisfied obviously, we proved the theorem for this case. 
Cases $S_{1}=X, S_{2}=Y$ and $S_{1}=Y, S_{2}=X$ and $S_{1}=X, S_{2}=S$ and $S_{1}=S, S_{2}=X$ are obvious consequences of Theorem 3

Consider the case, where the symbol of $L$ has exactly two different factors, that is $L$ is in the normalized form (2). There are only two cases to consider: $S_{1}=X, S_{2}=Y$, and $S_{1}=Y, S_{2}=X$.

Straightforward computations shows that the equality $H_{1} \circ H_{2}=G_{2} \circ G_{1}$, where $\operatorname{Sym}\left(H_{1}\right)=X$ and $\operatorname{Sym}\left(G_{1}\right)=Y$ implies that for some $a=a(x, y)$ and $b=b(x, y)$ we have $H_{2}=D_{x y}+a D_{x}+b D_{y}+a_{x}+a b=\left(D_{x}+b\right) \circ\left(D_{y}+a\right)$, while $G_{1}=D_{y}+a$. Thus, $H_{2}$ has a factorization of the type $(X)(Y)$.

Similar computations shows that the equality $H_{1} \circ H_{2}=G_{2} \circ G_{1}$, where $\operatorname{Sym}\left(H_{1}\right)=Y$ and $\operatorname{Sym}\left(G_{1}\right)=X$ implies that for some $a=a(x, y)$ and $b=$ $b(x, y)$ we have $G_{2}=D_{x y}+a D_{x}+b D_{y}+b_{y}+a b=\left(D_{y}+a\right) \circ\left(D_{x}+b\right)$, while $H_{1}=D_{y}+a$. Thus, $H_{2}$ has a factorization of the type $(Y)(X)$.

Example 1 (Symbol $S X Y, S_{1}=X+Y, S_{2}=Y$ ). We found an operator with two factorizations $L=\left(D_{x}+D_{y}+x\right) \circ\left(D_{x y}+y D_{x}+y^{2} D_{y}+y^{3}\right)$ and $L=$ $\left(D_{x x}+D_{x y}+\left(x+y^{2}\right) D_{x}+y^{2} D_{y}+x y^{2}+2 y\right) \circ\left(D_{y}+y\right)$. Then $L$ has factorization $L=\left(D_{x}+D_{y}+x\right) \circ\left(D_{x}+y^{2}\right) \circ\left(D_{y}+y\right)$.

Example 2 (Symbol $X^{2} Y, S_{1}=X, S_{2}=Y$ ). We found an operator with two factorizations $L=\left(D_{x}+x\right) \circ\left(D_{x y}+y D_{x}+y^{2} D_{y}+y^{3}\right)$ and $L=\left(D_{x x}+\left(x+y^{2}\right) D_{x}+\right.$ $\left.x y^{2}\right) \circ\left(D_{y}+y\right)$. Then $L$ has factorization $L=\left(D_{x}+x\right) \circ\left(D_{x}+y^{2}\right) \circ\left(D_{y}+y\right)$.

Example 3 (Symbol $X^{2} Y, S_{1}=Y, S_{2}=X$ ). We found an operator with two factorizations $L=\left(D_{y}+x\right) \circ\left(D_{x x}+y D_{x}+y^{3}-y^{4}\right)$ and $L=\left(D_{x y}+x D_{x}+\right.$ $\left.y^{2} D_{y}+x y^{2}+2 y\right) \circ\left(D_{x}+y-y^{2}\right)$. Then $L$ has factorization $L=\left(D_{y}+x\right) \circ\left(D_{x}+\right.$ $\left.y^{2}\right) \circ\left(D_{x}+y-y^{2}\right)$.

Looking at the examples, one can notice that the factorizations into firstorder factors have the right and left factors exactly the same as they were in the initial, given factorizations. In fact, this will be always the case. Accordingly, we improve Theorem 4 proving the following one.

Theorem 5. A third-order bivariate operator $L$ has a first-order left factor $F_{1}$ and a first-order right factor $F_{2}$ with $\operatorname{gcd}\left(\operatorname{Sym}\left(F_{1}\right), \operatorname{Sym}\left(F_{2}\right)\right)=1$ if and only if $L$ has a factorization into three factors, the left one of which is exactly $F_{1}$ and the right one is exactly $F_{2}$.

The following diagram is an informal illustration of the statement of the theorem:

$$
\left(L=F_{1} \circ \ldots \wedge L=\ldots \circ F_{2}\right) \Longleftrightarrow L=F_{1} \circ \ldots \circ F_{2} .
$$

Proof. Let $L$ have the normalized form (11). Then by Theorem 4 if $L$ has a firstorder left factor $F_{1}$ and a first-order right factor $F_{2}\left(\operatorname{Sym}\left(F_{2}\right)\right.$ is co-prime with $\left.\operatorname{Sym}\left(F_{1}\right)\right)$, it has a factorization into first-order factors of the type $\left(S_{1}\right)(R)\left(S_{2}\right)$, where $R=\operatorname{Sym}_{L} /\left(S_{1} S_{2}\right)$. Theorem 1 implies that such factorization is unique, so we have some unique first-order LPDOs $T_{1}, T, T_{2}$ such that $L=T_{1} \circ T \circ T_{2}$, where $\operatorname{Sym}\left(T_{1}\right)=S_{1}, \operatorname{Sym}(T)=R, \operatorname{Sym}\left(T_{2}\right)=S_{2}$. This also means that there 
are factorization $L=T_{1} \circ\left(T \circ T_{2}\right)$ of the type $\left(S_{1}\right)\left(R S_{2}\right)$ and factorization $L=\left(T_{1} \circ T\right) \circ T_{2}$ of the type $\left(S_{1} R\right)\left(S_{2}\right)$. Since $S_{1}, R, S_{2}$ are pairwise coprime, by Theorem 1 such factorizations are unique. On the other hand we have initial factorizations that are factorizations of the same types. Thus, we have $F_{1}=T_{1}$ and $F_{2}=T_{2}$.

For $L$ that has the normalized form (2), the statement of the theorem is actually a subresult in the proof of Theorem 4 for this case.

Proposition 1. The condition $\operatorname{gcd}\left(S_{1}, S_{2}\right)=1$ in Theorems 4 and 5 cannot be omitted.

Proof. Hyperbolic case. Consider an equivalence class of (1) defined by $q=1$, $I_{1}=I_{2}=I_{5}=0, I_{3}=I_{4}=x-y$ of the invariants from Thereom 2. Using Theorem 3 one can verify that operators of the class have factorizations of the types $(S)(X Y)$ and $(X Y)(S)$ only.

Such equivalence class is not empty. For example, operator $A_{3}=D_{x x y}+$ $D_{x y y}+(x-y)\left(D_{x}+D_{y}\right)$ belongs to this equivalence class. Only the following two factorizations exist for $A_{3}: A_{3}=\left(D_{x y}+x-y\right)\left(D_{x}+D_{y}\right)=\left(D_{x}+D_{y}\right)\left(D_{x y}+x-y\right)$.

The non-hyperbolic case. Consider operator of Landau

$$
D_{x}^{3}+x D_{x}^{2} D_{y}+2 D_{x}^{2}+(2 x+2) D_{x} D_{y}+D_{x}+(2+x) D_{y},
$$

which has two factorizations into different numbers of irreducible factors:

$$
L=Q \circ Q \circ P=R \circ Q,
$$

for the operators $P=D_{x}+x D_{y}, \quad Q=D_{x}+1, \quad R=D_{x x}+x D_{x y}+D_{x}+(2+$ $x) D_{y}$. That is factorizations of the types $(X)(S X),(S X)(X)$ exist, while those of the type $(X)(S)(X)$ do not. Here we denote $S=X+x Y$.

Proposition 2. The statement of Theorem 5 is not always true for a general fourth-order hyperbolic operator.

Proof. For example, operator

$$
\begin{aligned}
& L=\left(D_{x}+D_{y}\right) \circ\left(D_{x} D_{y}\left(D_{x}+D_{y}\right)+x D_{x x}+\left(2-x^{2}\right) D_{x}+x D_{y}-2 x+x^{2}\right) \\
& =\left(D_{x}\left(D_{x}+D_{y}\right)^{2}-x D_{x}\left(D_{x}+D_{y}\right)+(x-2) D_{x}+(x-1) D_{y}+1\right) \circ\left(D_{y}+x\right) .
\end{aligned}
$$

The second factor in the first factorization has no factorization.

\section{Completely Reducible Operators}

Let $\langle L\rangle$ denote the left ideal generated by an operator $L \in K[D]$. Consider Linear Ordinary Differential Operators (LODOs). The ring of LODOs are the principal ideal domain and, therefore, the intersection of two principal ideals is again principle. Consequently, the least common multiple $(\mathrm{lcm})$ of two LODOs $L_{1}$ and $L_{2}$ can be defined uniquely as $L$ such that $\left.\langle L\rangle=\left\langle L_{1}\right\rangle \cap<L_{2}\right\rangle$. Since in the ring of LPDOs this is not the case, it was suggested 9$]$ to introduce the notion of a completely irreducible LPDO. 
Definition 1. [9] An LPDO $L$ is said to be completely irreducible, if it can be expressed as $\langle L\rangle=\left\langle L_{1}>\cap \ldots \cap<L_{k}>\right.$ for suitable irreducible LPDOs $L_{1}, \ldots, L_{k}$. In this case $L=\operatorname{lcm}\left\{L_{1}, \ldots, L_{k}\right\}$ by definition.

Theorem 6. [9] If an LPDO $L$ has right factors $L_{1}, \ldots, L_{k}$ and

$$
\operatorname{Sym}_{L}=\operatorname{lcm}\left(\operatorname{Sym}_{L_{1}}, \ldots, \operatorname{Sym}_{L_{k}}\right) \text {, }
$$

then $\langle L\rangle=<L_{1}>\cap \ldots \cap<L_{k}>$. If the factors $L_{1}, \ldots, L_{k}$ are irreducible, then $L$ is completely reducible via $L_{1}, \ldots, L_{k}$.

An additional piece of motivation is 9 the following. Let for an ideal $I \subset$ $K[D]$ denote by $V_{I} \subset K$ its space of solutions. Then for two ideals $I_{1}, I_{2} \subset K[D]$ we have 2122] $V_{I_{1} \cap I_{2}}=V_{I_{1}}+V_{I_{2}}$, which allows to reduce the solution problem of the partial differential equation corresponding to a completely reducible LPDO to ones of corresponding to its factors.

Notice that the properties of the existence of a right factor with certain symbol or a factorization of certain factorization type, and, therefore, irreducibility of factors are invariant under the gauge transformations. Consequently, an invariant description of the completely reducible operators is possible.

Consider a hyperbolic linear partial differential operator of third order in the normalized form (1). Consider all possible right factors of the operator, their symbols are

$$
X, Y, S, X Y, X S, Y S,
$$

where $S=X+q Y$. Let us list all the possibilities for $\left\{L_{1}, \ldots, L_{k}\right\}$ that $L_{i}$-s together satisfy (10). Notice that the number (of factors) $k$ is not fixed. However, by to Thereom 1 there is no more than one factorization of each factorization type. Thus, $L_{i} \neq L_{j}$ for $i \neq j$, and, therefore, $k \leq 6$.

I. $\{X, Y, S\}$;

II. $\{S X, S Y\},\{S X, X Y\},\{S Y, X Y\}$;

III. $\{X, S Y\},\{Y, S X\},\{S, X Y\}$ - the right factors are co-prime ;

IV. $\{X, Y, S X\},\{X, X Y, S Y\}, \ldots-$ the sets that contain as the subset one of the sets of the groups $I I I$ and $I$.

V. $\{S X, S Y, X Y\}$ - the only set that contain as the subset one of the sets of the groups $I I$ and does not belong to the group $I V$.

Theorem 4 allows us to avoid the consideration of the large group $I V$. Indeed, By Thereom 4 at least one of the second-order factors of the sets fail to be irreducible.

Now, when we rule out all cases but eight, using Theorem 3 it is easy to obtain sufficient conditions for LPDOs to be completely reducible with

$$
<L>=<L_{1}>\cap \ldots \cap<L_{k}>,
$$

where $\left\{\operatorname{Sym}\left(L_{1}\right), \ldots, \operatorname{Sym}\left(L_{k}\right)\right\}$ belong to $I$ or $I I$ or $I I I$ or $V$. We just combine certain conditions from Theorem 3 Further below we use notation $\bar{W}$ for an arbitrary operator with the principal symbol $W$.

It is of interest to consider instead an important particular case $q=1$. We collect the sufficient conditions for this case in the following theorem. 
Theorem 7. Given an equivalence class of (1) by $q=1$, and values $I_{1}, \ldots, I_{5}$ of the invariants from Theorem Q Operators of the class are completely reducible with

I. $\langle L>=<\bar{X}>\cap<\bar{Y}>\cap<\bar{S}>$ if

$$
\begin{aligned}
& \left(D_{y}+I_{1}\right) \circ\left(2 D_{x}+D_{y}\right)\left(I_{1}\right)=0, \\
& I_{2}=I_{1 x}-I_{3}, \\
& I_{3}=\left(I_{1 y}+2 I_{1 x}\right) / 3, \\
& I_{4}=-I_{2}+I_{3}, \\
& I_{5}=I_{1} I_{1 x}-2 I_{1} I_{3}-I_{1 x y} / 2 ;
\end{aligned}
$$

II. $\langle L>=<\overline{S X}>\cap<\overline{S Y}>$ if

$$
\begin{aligned}
& I_{2}=F_{1}(y-x), \\
& I_{3}=I_{4}=0, \\
& I_{5}=-1 / 2 I_{1 x y}+I_{2 y},
\end{aligned}
$$

where $F_{1}(y-x)$ is some function;

$<L>=<\overline{S X}>\cap<\overline{X Y}>$ if

$$
\begin{aligned}
& I_{1 x y}+I_{2 x}-I_{1} I_{1 y}-2 I_{1} I_{2}=0, \\
& I_{3}=0 \text {, } \\
& I_{4}=-I_{1 y}+I_{1 x}-3 I_{2}, \\
& I_{5}=I_{4 y}-I_{1 x y} / 2+I_{2 y} \text {; } \\
& <L>=<\overline{S Y}>\cap<\overline{X Y}>\text { if } \\
& I_{1 x y}-I_{1} I_{1 x}-I_{2 y}+I_{1} I_{2}=0, \\
& I_{3}=I_{1 y}-I_{1 x}+3 I_{2} \text {, } \\
& I_{4}=0 \text {, } \\
& I_{5}=I_{3 x}-1 / 2 I_{1 x y}-I_{2 x} ; \\
& \text { III. }<L>=<\bar{X}>\cap<\overline{S Y}>\text { if } \\
& -I_{3 x}+\left(I_{1 x} I_{1}+I_{1 x y}+I_{1 x x}\right) / 2=0, \\
& I_{2}=I_{1 x} / 2 \text {, } \\
& I_{4}=0 \text {, } \\
& I_{5}=-1 / 2 I_{1 x y}+I_{2} I_{1}+I_{2 y} ; \\
& <L>=<\bar{Y}>\cap<\overline{S X}>\text { if } \\
& -I_{4 y}-I_{1 y} I_{1} / 2+I_{1 x y} / 2+I_{1 y y} / 2=0, \\
& I_{2}=-I_{1 y} / 2 \text {, } \\
& I_{3}=0 \text {, } \\
& I_{5}=I_{2} I_{1}-I_{1 x y} / 2-I_{2 x} ;
\end{aligned}
$$




$$
\begin{gathered}
<L>=<\bar{S}>\cap<\overline{X Y}>\text { if } \\
-I_{3 y}+\left(I_{1 x y}+I_{1 x x}-I_{1} I_{1 y}-I_{1} I_{1 x}\right) / 2-I_{3 x}=0, \\
I_{2}=\left(I_{1 x}-I_{1 y}\right) / 2, \\
I_{4}=-I_{2}+I_{3}, \\
I_{5}=\left(I_{1} I_{1 x}-I_{1} I_{1 y}-I_{1 x y}\right) / 2-I_{1} I_{3} ; \\
V .<L>=<\overline{S X}>\cap<\overline{S Y}>\cap<\overline{X Y}>\text { if } \\
I_{1 x x}-I_{1 y y}=0, \\
I_{1 x x}-2 I_{1} I_{1 x}+2 I_{1 x y}-I_{1} I_{1 y}=0, \\
I_{2}=\left(I_{1 x}-I_{1 y}\right) / 3, \\
I_{3}=I_{4}=0, \\
I_{5}=-I_{1 x y} / 2+I_{2 y} .
\end{gathered}
$$

\section{Conclusions}

The paper is devoted to the case when one LPDO has several factorizations.

In Sec. 4 we proved that a third-order bivariate operator $L$ has a first-order left factor $F_{1}$ and a first-order right factor $F_{2}$ with $\operatorname{gcd}\left(\operatorname{Sym}\left(F_{1}\right), \operatorname{Sym}\left(F_{2}\right)\right)=1$ if and only if $L$ has a factorization into three factors, the left one of which is exactly $F_{1}$ and the right one is exactly $F_{2}$. Also it was shown that the condition $\operatorname{gcd}\left(\operatorname{Sym}\left(F_{1}\right), \operatorname{Sym}\left(F_{2}\right)\right)=1$ is essential, and that the analogous statement "as it is" is not true for LPDOs of order four. However, other generalizations may be possible.

The proof for the hyperbolic case was done using invariants' methods. This is a nice and easy way to prove the things since the expressions for the necessary and sufficient conditions of the existence of factorizations of a given type are already known. It was the form of the conditions, that allowed us to make initial hypothesis that were proved later to be true in Sec. 4. However, some other method is required for generalizations to higher order LPDOs.

In Sec. 5 we considered the case, where one LPDO has two or more several factorizations of certain types. Most of the cases were ruled out from the consideration due to the results of Sec. 4 The explicit formulae for the sufficient conditions for the complete reducibility of an LPDO were found for the case $p=1$ (which is the case where the symbol of $L$ has constant coefficients only).

Acknowledgments. The author was supported by the Austrian Science Fund (FWF) under project DIFFOP, Nr. P20336-N18.

\section{References}

1. Tsarev, S., Shemyakova, E.: Differential transformations of parabolic second-order operators in the plane. Proceesings Steklov Inst. Math. (Moscow) (2009) see also electronically http://arxiv.org/abs/0811.1492. 
2. Tsarev, S.: Generalized laplace transformations and integration of hyperbolic systems of linear partial differential equations. In: ISSAC '05: Proceedings of the 2005 international symposium on Symbolic and algebraic computation, New York, NY, USA, ACM Press (2005) 325-331

3. Tsarev, S.: Factorization of linear partial differential operators and darboux' method for integrating nonlinear partial differential equations. Theo. Math. Phys. 122 (2000) 121-133

4. Anderson, I., Juras, M.: Generalized Laplace invariants and the method of Darboux. Duke J. Math. 89 (1997) 351-375

5. Anderson, I., Kamran, N.: The variational bicomplex for hyperbolic second-order scalar partial differential equations in the plane. Duke J. Math. 87 (1997) 265-319

6. Athorne, C.: A $z \times r$ toda system. Phys. Lett. A. 206 (1995) 162-166

7. A.V. Zhiber, S.S.: Integrals, solutions and existence of the laplace transformations for a linear hyperbolic system of equations. Math. Notes 74(6) (2003) 848-857

8. Startsev, S.: Cascade method of laplace integration for linear hyperbolic systems of equations. Mathematical Notes 83 (2008)

9. Grigoriev, D., Schwarz, F.: Factoring and solving linear partial differential equations. Computing 73(2) (2004) 179-197

10. Grigoriev, D., Schwarz, F.: Generalized loewy-decomposition of d-modules. In: ISSAC '05: Proceedings of the 2005 international symposium on Symbolic and algebraic computation, New York, NY, USA, ACM (2005) 163-170

11. Grigoriev, D., Schwarz, F.: Loewy decomposition of third-order linear pde's in the plane. In: ISSAC '08: Proceedings of the 2005 international symposium on Symbolic and algebraic computation, New York, NY, USA, ACM (2008) 277-286

12. Li, Z., Schwarz, F., Tsarev, S.P.: Factoring systems of linear pdes with finitedimensional solution spaces. J. Symb. Comput. 36(3-4) (2003) 443-471

13. Li, Z., Schwarz, F., Tsarev, S.: Factoring zero-dimensional ideals of linear partial differential operators. In: ISSAC '02: Proceedings of the 2002 international symposium on Symbolic and algebraic computation, New York, NY, USA, ACM Press (2002) 168-175

14. Tsarev, S.P.: An algorithm for complete enumeration of all factorizations of a linear ordinary differential operator. In: ISSAC '96: Proceedings of the 1996 international symposium on Symbolic and algebraic computation, New York, NY, USA, ACM (1996) 226-231

15. Shemyakova, E., Winkler, F.: Obstacles to the Factorization of Linear Partial Differential Operators into Several Factors. Programming and Computer Software 33(2) (2007) 67-73

16. Blumberg, H.: Über algebraische Eigenschaften von linearen homogenen Differentialausdrücken. PhD thesis, Göttingen (1912)

17. Shemyakova, E., Mansfield, E.: Moving frames for laplace invariants. Proceedings of ISSAC'08 (The International Symposium on Symbolic and Algebraic Computation) (2008) 295-302

18. Shemyakova, E., Winkler, F.: On the invariant properties of hyperbolic bivariate third-order linear partial differential operators. LNAI 5081 (2007)

19. Shemyakova, E.: On the invariant properties of non-hyperbolic third-order linear partial differential operators. Conferences on Intelligent Computer Mathematics $\mathbf{5 6 2 5}$ (2009)

20. Shemyakova, E., Winkler, F.: A full system of invariants for third-order linear partial differential operators in general form. Lecture Notes in Comput. Sci. 4770 (2007) 360-369 
21. Cassidy, P.: Differential algebraic groups. Amer. J. Math. 94 (1972) 891-95

22. Sit, W.: Typical differential dimension of the intersection of linear differential algebraic groups. J. Algebra 32(3) (1974) 476-487 